\title{
RGB1 regulates grain development and starch accumulation through transactivation of OsNF-YB1 on OsYUC11-mediated auxin biosynthesis
}

\author{
Dongping Zhang ${ }^{1}$, Minyan zhang ${ }^{2}$, and Jiansheng Liang ${ }^{1}$ \\ ${ }^{1}$ Southern University of Science and Technology \\ ${ }^{2}$ Yangzhou University
}

June 15, 2020

\begin{abstract}
RGB1, a subunit of heterotrimeric G protein, plays important roles in regulating grain size and weight of rice. However, the molecular mechanisms underlying controlling grain filling process by $\mathrm{G}$ protein are still largely unclear. In the present study, we show that, RGB1 controls not only the grain size, but the grain filling process. Knock-down of RGB1 significantly delayed grain development and reduced starch accumulation and grain weight, which was closely related to the delayed and lower expression of genes encoding sucrose metabolism and starch biosynthesis related enzymes during grain filling stage. Suppression of RGB1 expression also resulted in the lower auxin content in grains, which was correlated with the lower expression of OsNF-YB1 and OsYUC11 during grain filling stage. Further biochemical evidence showed that OsYUC11 expression was under control of OsNFYB1 by its interaction with promoter of OsYUC11. Taken together, we propose that RGB1 controls rice grain development and grain filling process by changing auxin homeostasis in endosperm cells, and OsNF-YB1, which acts as a key downstream effector of RGB1, interacts directly with the promoter of OsYUC11 and promotes the transactivation of OsYUC11, thereby regulating auxin biosynthesis and starch accumulation and grain size.
\end{abstract}

\section{Acknowledgements}

We would like to thank Prof. Cunxu Wei and Dr. Juan Wang at Yangzhou University for the fruitful discussions and valuable suggestions on this study.

\section{Summary statement}

RGB1 regulates grain development and starch biosynthesis by controlling auxin synthesis, of which RGB1 positively regulates the expression of $O s Y U C 11$, a key gene responsible for auxin synthesis in rice endosperm cells, through activating the interaction of OsNF-YB1, a nuclear transcript factor, with OsYUC11 .

RGB1 regulates grain development and starch accumulation through transactivation of OsNFYB1 on $O s Y U C 11$-mediated auxin biosynthesis in rice endosperm cells

\begin{abstract}
RGB1, a subunit of heterotrimeric G protein, plays important roles in regulating grain size and weight of rice. However, the molecular mechanisms underlying controlling grain filling process by $\mathrm{G}$ protein are still largely unclear. In the present study, we show that, RGB1 controls not only the grain size, but the grain filling process. Knock-down of $R G B 1$ significantly delayed grain development and reduced starch accumulation and grain weight, which was closely related to the delayed and lower expression of genes encoding sucrose metabolism and starch biosynthesis related enzymes during grain filling stage. Suppression of $R G B 1$ expression also resulted in the lower auxin content in grains, which was correlated with the lower expression
\end{abstract}


of OsNF-YB1 and OsYUC11 during grain filling stage. Further biochemical evidence showed that OsYUC11 expression was under control of OsNF-YB1 by its interaction with promoter of OsYUC11. Taken together, we propose that RGB1 controls rice grain development and grain filling process by changing auxin homeostasis in endosperm cells, and OsNF-YB1, which acts as a key downstream effector of RGB1, interacts directly with the promoter of OsYUC11 and promotes the transactivation of OsYUC11, thereby regulating auxin biosynthesis and starch accumulation and grain size.

Keywords: auxin, grain filling, OsNF-YB1, OsYUC11, RGB1, rice, starch biosynthesis

\section{INTRODUCTION}

The rice grain weight is determined by both the grain sink size (numbers and size of endosperm cells) and the physiological and biochemical activities (sucrose metabolism and starch biosynthesis) of the endosperm cells for cereals (Liang et al., 2001). The rice grain is composed of the embryo and the endosperm, which is enclosed by a thin seed coat and covered by the spikelet hull (the husk), which physically restricts the size of caryopsis. The endosperm, which is the major sites storing starches and other nutritious compounds, occupies the bulk of the caryopsis. In this sense, the size and weight of the mature grain are mainly determined by the shape and size of the spikelet hull and the filling degrees of caryopsis (mainly endosperm), both of which varied greatly and controlled by different genetic and environmental factors. Rapid advances in rice functional genomics and the advent of next generation sequencing technologies have led to the recent cloning of a series of quantitative trait loci (QTLs)/genes to control grain size (length, width, thickness) and grain weight (See: Reviewed by Li et al., 2018; Table S1). However, the underlying molecular mechanism regulating the grain development and grain filling processes remains elusive.

Heterotrimeric $\mathrm{G}$ protein (hereafter $\mathrm{G}$ protein)-mediated signal transduction pathway is considered as one of the most important signaling mechanisms that regulates various important physiological and molecular processes both in animals and plants (Jones \& Assmann, 2004; Urano et al., 2013; Urano \& Jones, 2014 ). In general, $G$ protein is composed of three subunits, $G \alpha, G \beta$ and $G \gamma$. Several researches showed that the $\mathrm{G} \beta$ and $\mathrm{G} \gamma$ works as a single functional subunit (dimer) to regulate organ size (Urano et al., 2013). Rice genome encodes one G $\alpha$ (RGA1), one G $\beta$ (RGB1), and five Grs (RGG1, RGG2, GS3, DEP1/qPE9-1 and GGC2), and evidence have shown all these $\mathrm{G}$ protein subunits play important roles in regulating rice grain size ( Ashikari et al., 1999; Fujisawa et al., 1999; Huang et al., 2009; Zhou et al., 2009; Mao et al., 2010; Trusov et al., 2012; Liu et al., 2018; Sun et al., 2018; Zhang et al., 2019). RGA1 and two Gr subunits, GGC2 and DEP1/qPE9-1, positively regulate rice grain size, whereas other three Gr subunits, RGG1, RGG2 and GS3, play opposite roles in grain size regulation ( Oki et al., 2005; Huang et al., 2009; Zhou et al., 2009; Mao et al., 2010; Liu et al., 2018; Sun et al., 2018). Interestingly, bothRGB1 -knocking down lines and $R G B 1$-overexpressing lines show smaller rice grain size as compared with the wildtype (Utsunomiya et al., 2011; Liu et al., 2018; Sun et al., 2018). A possible explanation is that $\mathrm{G} \gamma$ and $\mathrm{G} \beta$ function as a dimer and their roles in regulating rice grain size depend on competitively coupling of G $\beta$ with different $\mathrm{G} \gamma$ subunits. Of course, other factors cannot be ruled out, such as MADS-domain transcription factor, BR transcription factor, BES1, etc (Liu et al., 2018; Zhang et al., 2018).

Although $\mathrm{G}$ proteins play important roles in regulating grain size, the molecular mechanisms underlying controlling grain filling process by $G$ protein are still largely unclear. In the present study, we provide both genetic and biochemical evidence to show that, RGB1 controls not only the grain size, but the grain filling process as well. Knock-down of $R G B 1$ significantly delayed grain development and reduced starch accumulation and grain weight, which is closely related to the delayed and lower expression of genes encoding sucrose metabolism and starch biosynthesis related enzymes during grain filling stage. The lower auxin levels in the grains of the $R G B 1-k$ nocking down lines are due to the lower expression of $O s Y U C 11$ controlled by OsNF-YB1, a transcript factor, and NAA application can partially recovered the grain filling through stimulating the expression of sucrose metabolism and starch biosynthesis related genes.

\section{MATERIALS AND METHODS}

Generations of knock-down mutants using RNA interfering technology 
To generate RGB1 RNAi transgenic lines, a gene fragment of RGB1 was amplified from Nipponbare cDNA. A hairpin structure with two inverted repeat fragments was subsequently constructed and transferred into the plant binary vector pTCK303, expressed under the control of the maize ubiquitin promoter (Wang et al., 2004). The construct was transformed into the Agrobacterium strain EHA105 and used for plant transformation as described previously (Chen et al. , 2014). The DNA fragments embracing the targets of the plant transformants were amplified for sequencing.

\section{Plant growth conditions and treatments}

The experiment was carried out at the farm of Yangzhou University $\left(32^{\circ} 30^{\prime} \mathrm{N}, 119^{\circ} 25^{\prime} \mathrm{E}\right)$ during the rice (Oryza sativa) growing season, and in Hainan in winter seasons. At the heading stage, 200 uniformly growing and headed panicles (1-2 panicles per plant) were chosen, and spikelets on the selected panicles with the same flowering date were labeled for each cultivar/line. The flowering date and position of each spikelet on the labeled panicles were recorded. Approximately 30 labeled panicles were sampled each time from flowering to maturity. Half of the sampled grains were frozen in liquid nitrogen for at least 2 min and then stored at $-80^{\circ} \mathrm{C}$ for further analyses. The other half of the grains were dried at $80^{\circ} \mathrm{C}$ for approximately $72 \mathrm{~h}$ to a constant weight and used for starch analyses.

For exogenous NAA application, rice plants growing in the plastic pots (three hills per pot) under open field conditions were used. Each pot $(0.6 \mathrm{~m}$ in height, $0.5 \mathrm{~m}$ and $0.3 \mathrm{~m}$ in diameter at the top and bottom, respectively) was filled with sandy loam soil that contained the same nutrient contents as the field soil. The sowing date and cultivation were the same as those for the field experiment. After flowering, $10 \mu \mathrm{M} \mathrm{NAA}$ was sprayed at a rate of $5 \mathrm{ml}$ per pot on the top of the plants (spikes) every 5 days until 30 DAF. The solutions contained ethanol and Tween-20 at final concentrations of $0.1 \%(\mathrm{v} / \mathrm{v})$ and $0.01(\mathrm{v} / \mathrm{v})$, respectively. Control plants were sprayed with the same volume of deionized water containing the same concentrations of ethanol and Tween-20.

\section{Gene expression analysis}

Total RNA was extracted from the grains or young panicle using the RNAprep Pure Plant Kit (Tiangen). The HiScript II Q Select RT SuperMix (Vazyme) was used for cDNA synthesis. The transcript levels of each gene were measured by qRT-PCR using a 7500 Real-Time PCR System (ABI) with PowerUp SYBRß Green Master Mix (Thermo Fisher Scientific). Gene expression was quantified during the logarithmic phase using the expression of the housekeeping gene Ubq (LOC_Os03g13170) as an internal control. Three biological replicates were performed for each experiment.

\section{Hormone quantification}

The IAA level was determined by Zoonbio Biotechnology Co., Ltd. $0.5 \mathrm{~g}$ samples were ground in a pre-cooled mortar that contained $5 \mathrm{~mL}$ extraction buffer composed of isopropanol/hydrochloric acid. The extract was shaken at $4^{\circ} \mathrm{C}$ for $30 \mathrm{~min}$. Then, $10 \mathrm{~mL}$ dichloromethane was added, and the sample was shaken at $4^{\circ} \mathrm{C}$ for 30 min and centrifuged at 13,000 rpm for $5 \mathrm{~min}$ at the same temperature. The organic phase was dried under $\mathrm{N}_{2}$ gas and the pellet dissolved in $150 \mu \mathrm{L}$ methanol ( $0.1 \%$ methane acid) and filtered with a $0.22-\mu \mathrm{m}$ filter membrane. The purified product was then subjected to high-performance liquid chromatography-tandem mass spectrometry (HPLC-MS/MS) analysis. HPLC analysis was performed using a ZORBAX SB-C18 (Agilent Technologies) column $(2.1 \mathrm{~mm} \times 150 \mathrm{~mm} ; 3.5 \mathrm{~mm})$. The mobile phase A solvents consisted of methanol $/ 0.1 \%$ methanoic acid, and the mobile phase B solvents consisted of ultrapure water $/ 0.1 \%$ methanoic acid. The injection volume was $2 \mu \mathrm{L}$. MS conditions were as follows: the spray voltage was $4500 \mathrm{~V}$; the pressure of the air curtain, nebulizer, and aux gas were 15, 65, and 70 psi, respectively; and the atomizing temperature was $400^{\circ} \mathrm{C}$.

\section{Protein blot analysis}

Rice grains (from 5 to 25 DAF) were homogenized in TEDM buffer (20 mM Tris/HCl, pH 7.5, 1 mM DTT, 5 $\mathrm{mM}$ EDTA, and $10 \mathrm{mM} \mathrm{MgCl} 2$ ) containing a complete protease inhibitor cocktail (Roche). The homogenate was centrifuged at $6,000 \mathrm{~g}$ for $10 \mathrm{~min}$ at $4^{\circ} \mathrm{C}$ to remove cellular debris, and the supernatant was clarified 
by centrifugation at $12,000 \mathrm{~g}$ for $10 \mathrm{~min}$ at $4^{\circ} \mathrm{C}$. The soluble proteins were separated by SurePAGE (4-12\%, Genscript) and blotted onto PVDF membrane (Millipore). Anti-HSP82 antibodies were used as a loading control (Beijing Protein Innovation).

\section{Transcriptome sequencing}

For transcriptome sequencing, total RNA was extracted from the grains at 5 and 10 DAF, using the RNAprep Pure Plant kit (Tiangen). RNA integrity and quantity were determined with an Agilent 2100 Bioanalyzer according to the manufacturer's protocol. Enrichment of mRNA from total RNA, complementary DNA synthesis, and construction of the library were performed at Vazyme Biotech. The total libraries were sequenced using an Illumina HiSeq X Ten platform. The raw reads were filtered by removing reads with adapters, reads in which unknown bases were $>5 \%$, and low-quality reads. The FASTQC program was used to assess the quality of the clean reads, which were then aligned to the rice reference genome Release 7 of the MSU Rice Genome Annotation Project using TOPHAT v.2.1.0 (Trapnell et al., 2012). CUFFLINKS v.2.1.1 was then used to normalize and estimate the gene expression level according to fragments per kilobase of transcript per million reads (FPKM, Mortazavi et al., 2008). The differentially expressed genes (DEGs) were also calculated using CUFFLINKS v.2.1.1 at a significance level of $\mathrm{q}<0.05$ and the absolute value of $\log 2$ ratio [?] 1.

\section{Electrophoresis mobility shift assay (EMSA)}

The flag-OsNF-YB1 protein was expressed using Gzl Custom Cell Free Protein Expression Kit (GZL bioscience). DNA probes in a length of 59 nt were commercially synthesized by GENEWIZ Biological Technology and labeled with an EMSA Probe Biotin Labeling Kit (Beyotime, Cat No. GS008,). DNA binding was performed in a $10 \mathrm{~L}$ reaction volume containing EMSA/Gel-shift binding buffer (Beyotime), 2 nmol biotin-labeled probe, $5 \mathrm{nmol}$ purified recombinant protein. Non-labeled DNA oligos were used as competitor. Recombinant protein was pre-incubated with the EMSA/Gel- shift binding buffer for 20 min at 25 $\operatorname{deg} \mathrm{C}$ prior to the addition of the biotin-labeled probe and further incubated at $25 \operatorname{deg} \mathrm{C}$ for $20 \mathrm{~min}$. A $6 \%(\mathrm{~W} / \mathrm{V})$ polyacrylamide gel was pre-run for $30 \mathrm{~min}$, and then the binding reaction is subjected to gel electrophoresis. The DNA probes were then transferred to a charged nylon membrane (Beyotime), detected by streptovidin-HRP (Beyotime), and finally visualized using the enhanced chemiluminescence (Pierce).

\section{Statistical analysis}

Data are presented as mean +- SD. SPSS16.0 software was used for all statistical analysis. Statistical significance was determined by independent biological samples student's t-test for comparison of two groups and one way ANOVA for comparison of three or more groups. Differences were considered statistically significant when $\mathrm{P}<0.05$. P-values are indicated by "**" when $\mathrm{P}<0.05$ or "**" when $\mathrm{P}<0.01$.

\section{RESULTS}

\section{$R G B 1$ positively regulates both grain size and grain filling}

Previous studies have showed that $R G B 1$ play important roles in regulating growth and development of rice plants (Utsunomiya et al., 2011; Sun et al ., 2018). However, it remains largely unknown how the grain development and grain filling is regulated by $R G B 1$. To investigate the molecular mechanisms of RGB1 regulation on grain development and grain filling, we used Wuyujing 8 (hereafter $W Y J 8$ ) as background, to generate two RGB1 RNA-interfered transgenic lines (hereafter RGB1Ri-5 and RGB1Ri-6). Compared with WYJ8, the grain RGB1 protein levels were significantly lower in RGB1Ri-5 and RGB1Ri-6 lines, measured by western blot assay using a RGB1-specific antibody (Figure 1a). The final grain size, including the length, width, and thickness of grains, of two transgenic rice lines with suppressed expression of $R G B 1$ were markedly reduced, and consequently, the final grain weight reduced significantly, as compared with WYJ8 (Figure 1b1f), which was very similar to the phenotype observed in several other reports (Utsunomiya et al., 2011; Sun et al., 2018). Furthermore, the grain starch content of RGB1Ri lines was also lower than that of WYJ8 (Figure 1g). We further tracked the caryopsis development and dry matter accumulation after flowering, and the caryopsis size and grain weight of WYJ8 increased rapidly and maximal values were reached at 
25 DAF. However, the increase of caryopsis size was obviously delayed and the dry matter accumulation of grains much slowly during the early stage of grain filling of $R G B 1 R i$ lines (Figure $1 \mathrm{~h}$ and $1 \mathrm{i}$ ). These results implied that $R G B 1$ might also be involved in regulating the grain filling processes as well as the grain size.

$R G B 1$ enhances the expression of genes associated with sucrose metabolism and starch biosynthesis

To investigate how $R G B 1$ regulates the grain development and starch biosynthesis, the expression profiles of genes in caryopsis of WYJ8 and RGB1Ri-5 line at 5 and 10 DAF were analyzed, using the RNA-seq assay. Approximately 3216 and 3363 genes that were up- or down-regulated, respectively, in RGB1Ri5 as compared with $W Y J 8$, were identified at 5 DAF. The numbers of up-regulated genes at 10 DAF decreased to 2832 and those of down-regulated genes increased to 3398, respectively (Figure S1a). The Kyoto Encyclopedia of Genes and Genomes (KEGG) pathway enrichment analysis showed that these differently expressed genes (DEGs) were significantly enriched for "sucrose metabolism and starch biosynthesis" and "plant hormone transduction" (Figure S1b), suggesting that RGB1 may regulate sucrose metabolism and grain starch synthesis through manipulating hormone homeostasis and/or hormone-mediated signaling.

To validate above hypothesis, we analyzed the expression patterns of DEGs for sucrose metabolism and starch synthesis (29 genes) and grain development regulation (17 genes). The results indicated that the expression of about $70 \%$ of the sucrose metabolism and starch biosynthesis-related genes and all the grain development-related genes were up-regulated in WYJ8, as compared with that inRGB1Ri-5 line (Figure 2a). These results were consistent with our observation that $R G B 1$ positively regulate grain filling and starch accumulation. We further analyzed the dynamic expression patterns of these genes in grains of WYJ8 and RGB1Ri lines during grain filling period using both real-time quantitative PCR and western blotting assay. As shown in Figure 2b, the dynamic expression patterns of all twelve sucrose metabolism and starch biosynthesis-related genes could be roughly divided into three groups. OsAGPS2a, OsAGPS2b , OsISA2, OsSSSI , OsSSSIIa and OsPUL belonged to the first group, of which their transcript levels rapidly increased after flowering, and reached their maximal levels at $20 \mathrm{DAF}$, and then decreased both in WYJ8 and in $R G B 1 R i$ lines. However, the expression levels of these six genes in grains of WYJ8 were higher than in $R G B 1 R i$ lines, especially during early stage of grain filling. The second group included OsISA1, OsBE1 and $O s B E I I b$, and the transcript levels of these three genes increased in grains of all genotypes, but the time reached maximal levels was different between genotypes. The maximal transcript levels of these genes in WYJ8grains were detected at $10 \mathrm{DAF}$, which was much earlier than those ofRGB1Ri lines. OsAGPL2, OsSSSIII a and OsGBSSIgenes belonged to the third group, of which the transcript level in grains of WYJ8 line was higher at the early stage of grain filling, whereas the transcript levels in grains of $R G B 1 R i$ lines increased rapidly after $10 \mathrm{DAF}$ and kept very higher levels thereafter. The western blotting results were well consistent with the RT-PCR results, of which the protein level of OsSSSIIa, OsBEI, OsBEIIb, OsSSSI and OsGBSSI were much lower in grains of RGB1Ri-5 line at the early stage of filling (Figure 2c).Taken together, these results indicate that $R G B 1$ play key roles in controlling grain filling through its effects on the expression of genes encoding sucrose metabolism and starch biosynthesis-related enzymes, especially at the early stage of grain filling.

\section{$R G B 1$ stimulates accumulation of IAA by regulating the expression of auxin biosynthesis- related genes}

It is well known that grain development and sucrose metabolism and starch biosynthesis are highly regulated by both the genetic and the environmental cues. Plant hormones are involved in the regulation of many aspects of grain development and sucrose metabolism and starch biosynthesis (Tang et al., 2009; Yang \& Zhang, 2010; Hu, et al., 2013; Zhang et al., 2014; Zuo \& Li, 2014). Our previous study and other research groups' results clearly showed that auxin plays important roles in these processes (Zhao, 2008; Ghorbani et al., 2011; Abu-Zaitoon et al. 2012; Zhao et al. 2013; Tamaki et al., 2015; McAdam et al., 2017). However, the molecular basis of auxin accumulation in rice grains is largely unclear. Based on the results of RNA-seq assay and the KEGG pathway enrichment analysis, we found that there were 77 DGEs involved in auxin biosynthesis and metabolism, transport, and signaling during grain filling period (Figure 
S2). Among them, six auxin biosynthesis genes were up-regulated in WYJ8 grains at $10 \mathrm{DAF}$, as compared with those in $R G B 1 R i-5$ line (Figure $3 \mathrm{a}$ ), implying that $R G B 1$ positively regulates sucrose metabolism and starch biosynthesis probably through controlling IAA biosynthesis in grains.

To validate this hypothesis, we measured the endogenous IAA content of grains during grain filling period. The results showed that the $W Y J 8$ grain IAA content increased almost linearly after flowering and reached its maximal value at $20 \mathrm{DAF}$, whereas those of $R G B 1 R i$ lines increased much slowly, especially at the early stage of grain development, and kept much lower levels throughout all experimental period (Figure $3 \mathrm{~b}$ ). Application of NAA after flowering accelerated starch accumulation and enhanced the final grain weight significantly of $R G B 1 R i-5 / 6$ lines (Figure 3c-3d). NAA application also stimulated the expression of OsSSSI, OsSSSIIa, OsGBSSI,OsBEIIb and OsAGPS2a, but had no effects on the expression of other seven genes in Figure 2, as compared with the no-NAA treated RGB1Ri-5 line (Figure 3d). All these indicate positive correlations between IAA and sucrose metabolism and starch biosynthesis and between $R G B 1$ expression and grain IAA level. So, it is reasonable to assume that $R G B 1$ stimulates the grain filling and sucrose metabolism and starch biosynthesis largely through influencing auxin biosynthesis.

In higher plants, the de novo IAA biosynthesis is viaTAR/YUCCA pathway. Our RNA-seq analysis results revealed that several auxin biosynthesis genes, including $Y U C s$ and TARs, were down-regulated in grains of $R G B 1 R i$ lines either at $5 \mathrm{DAF}$ or $10 \mathrm{DAF}$ (Figure 3a). There is evidence that IAA plays several distinct roles at different stages of endosperm development (Figueiredo \& Kohler, 2018; Basunia \& Nonhebel, 2019). These differing roles require strong localized control of IAA biosynthesis by specific enzymes. According to the public available expression data, three genes, OsYUC9, OsTAR1 and OsYUC11, are specifically and highly expressed in rice endosperm cells (Figure 4a) .Our qRT-PCR results showed that the transcript levels of all three genes increased at the early filling stage of wildtype grains, whereas those in $R G B 1 R i$ lines kept lower at the same stage of grain filling and increased till 15 DAF (Figure 4b and Figure S3a), suggesting their involvement in IAA biosynthesis in grains. However, among three genes, only the expression of OsYUC11 was significantly correlated to the grain IAA levles (Figure 4c and Figure S3b). If we considered the temporal expression patterns of $O s Y U C 11$ combined with the results of the initiation of rapid accumulation of IAA and starch in grains of both wildtype and $R G B 1 R i$ lines (Figure $1 \mathrm{i}$ and $3 \mathrm{~b}$ ), it is reasonable to assume that various auxin biosynthesizing genes may control auxin production in different organs/tissues and the endosperm-specific $O s Y U C 11$ gene is the most important one that is responsible for auxin biosynthesis of grains during filling stage, and that $R G B 1$ stimulates auxin accumulation through enhancing the expression of $O s Y U C 11$ gene in grains.

\section{RGB1 regulates expression of $O s Y U C 11$ probably through $O s N F-Y B 1$}

The results above showed that RGB1 regulates grain development and sucrose metabolism and starch accumulation probably through stimulating the expression of endosperm-specific OsYUC11, which enhances auxin biosynthesis in endosperm cells. However, how RGB1 regulates the expression of OsYUC11 is still elusive. In the present experiment, we first analyzed the promoter of OsYUC11 that might bind and regulate OsYUC11 expression using PlantPAN 2.0 database. The results showed that there were 305 putative transcript factors which might bind to the promoter of OsYUC11 (Figure 5a; Table S2). Among them, four transcript factors, $O s 08945110, O s N F-Y B 1, O s A B I 5$ and $O s E I L 4$, were both co-expressed with $O s Y U C 11$ and down-regulated in RGB1Ri line on 5 and 10 DAF (Figure $5 \mathrm{a}$ and $5 \mathrm{~b}$ ), implying that the expression of OsYUC11 might be regulated by these four transcript factors. We further compared the temporal expression patterns of these four transcript factors in grains of wildtype and $R G B 1 R i$ lines and the results showed that only the transcript levels of $O s N F-Y B 1$ increased rapidly and were higher in the wildtype grains than those in $R G B 1 R i$ lines, especially at the rapid stage of grain filling (Figure 5c), which is well consistent with those of starch accumulation and grain IAA content (Figure 1i and 3b and 3c). Furthermore, the transcript levels of $O s N F-Y B 1$ were positively related to those of $O s Y U C 11$ (Figure 5d). It is reasonable to assume that $O s N F-Y B 1$ play a key role in controlling $O s Y U C 11$ expression, and finally the auxin content in grains. To verify this assumption, we also conducted an EMSA assay and the results clearly showed that OsNF-YB1 was able to bind the promoter of OsYUC11 (Figure 5e). Based on above results, we proposed that the 
expression of OsYUC11 in rice grains was under the control of OsNF-YB1 .

\section{Discussion}

The grain size and weight of rice are complex traits, which involves in grain development and filling process (mainly the sucrose metabolism and starch biosynthesis), and is controlled by both the genetic and the environmental factors. In last decades, several research groups have provided genetic and molecular evidence that grain size and filling processes are controlled by many genes (see review by Zuo \& Li, 2014; Sun et al., 2018). All these researches provide rice breeders opportunities to improve rice grain yield by manipulating these genes. However, it still has a long way to go before understanding the complex regulatory networks involving grain development and filling processes. The heterotrimeic $\mathrm{G}$ protein (hereafter $\mathrm{G}$ protein) is well known to play important roles in plant growth and development (Botella, 2012; Sun et al., 2018). Recently, several rice genes/QTLs encoding G protein subunits have been shown to control grain size and shape of rice, including: qPE9-1/DEP1 , GS3 , RGB1 and $R G A 1$ (Oki et al., 2005; Huang et al., 2009; Zhou et al. , 2009; Mao et al., 2010; Liu et al., 2018; Miao et al., 2018; Sun et al., 2018).qPE9-1/DEP1 encoding a Gr subunit, positively regulates grain size (Huang et al., 2009; Zhou et al., 2009); GS3, another Gr subunit, negatively regulate grain size and shape (Mao et al., 2010; Sun et al., 2018); RGB1 and RGA1, encoding G $\beta$ and $\mathrm{G} \alpha$ subunit, respectively, positively regulates grain size and shape (Fujisawa et al. , 1999; Utsunomiya et al., 2011; Sun et al., 2018). However, the functions and molecular mechanisms of G protein to regulate grain development and starch biosynthesis are largely unknown.

Rice grain filling is a very complicated process that involves photoassimilate translocation from photosynthetic sources (i.e., leaves and stem-sheaths), sucrose degradation, transmembrane transport and starch synthesis in the grains (Liang et al., 2001; Lü et al., 2008; Tang et al., 2009). Approximately twenty enzymes/proteins have been reported to be involved in these biochemical processes (Zhu et al. , 2003; Tetlow et al., 2004; Ohdan et al., 2005). The results of our lab and several other groups showed that sucrose synthase (SUS), invertase (INV), ADP-glucose pyrophosphorylase (AGP), soluble starch synthase (SSS) and grannuel-bound starch synthase (GBSS), branching enzyme (BE) and debranching enzyme (DBE) play key roles in the regulation of sucrose metabolism and starch biosynthesis (Liang et al., 2001; Lü et al. , 2008; Tang et al., 2009; also see reviewed by Tetlow et al., 2004; Keeling \& Myers, 2010; Zeeman et al., 2010). The grain-filling process is also a highly regulated process in which both genetic and environmental factors are involved. It is well known that plant hormones play important roles in grain development and filling process (Liang et al. , 2001; Yang et al., 2006; Tang et al., 2009; see also reviewed by Basunia \& Nonhebel, 2019). Understandably, rice grain size, not like the wheat grains, is physically limited by the spikelet hull, of which the final size is almost determined upon flowering. In this sense, the endosperm cell proliferation and elongation as well as the accumulation of starch and other storage compounds after flowering are of crucial importance for final grain yield and quality. Our results showed that grain size, including the length, width and thickness of grains reduced, and as a result, the final grain weight and starch content reduced significantly in $R G B 1$ knock-down lines (Figure 1). This reduction was mainly due to the delay of caryopsis development and the lower starch accumulation at the early stage of grain filling. As we known, the rice grain filling process is in fact the process of sucrose metabolism and starch biosynthesis occurred in endosperm cells, in which many enzymes/proteins are involved. So, it is expected that the expression levels of genes encoding enzymes catalyzing sucrose metabolism and starch biosynthesis during grain filling are closely related to the starch accumulation and grain weight. In $R G B 1$ knock-down lines, the expression of genes encoding sucrose metabolism and starch biosynthesis was either initiated later or much lower at the early filling stage, which can well explain the results observed here (Figure 1 and 2) and imply that suppression of RGB1 expression could down-regulate the expression of genes encoding enzymes catalyzing sucrose metabolism and starch biosynthesis. However, we still unknown the molecular mechanisms of $R G B 1$ regulation on the expression of these genes.

Recently, several research groups have reported that an accumulation of auxin immediately before the starch biosynthesis in rice grains (Abu-Zaiton et al., 2012). Furthermore, application of exogenous auxin also had

a positive effect on starch accumulation. These results suggested that auxin may involve in the regulation of 
starch biosynthesis in rice grains. RNA-seq assay and auxin quantitation also showed a great difference in the expression of auxin biosynthesis related genes and IAA contents in grains between $R G B 1$ knocking down lines and wildtype during filling stage. The expression of endosperm-specific genes for auxin biosynthesis was down-regulated and endogenous IAA content significantly reduced in the grains of $R G B 1$ knocking down lines (Figure 3). The assumption that RGB1 involvement in the regulation of starch biosynthesis is through changing auxin homeostasis of grains was further validated according to the results of exogenous application of IAA on starch accumulation and the expression of sucrose metabolism and starch biosynthesis related genes during grain filling stage (Figure 3).

Auxin biosynthesis in higher plants is catalyzed by a large number of TARs (encoding tryptophan aminotransferase) and YUCs(encoding indole-3-pyruvate mono-oxygenases) with differing patterns of spatiotemporal expression, which allows for multiple roles. Different genes may be responsible for the auxin production in different time and/or in different tissues, and therefore, play various roles in regulating plant growth and development. In rice, tissue-specific expression of these genes showed that OsTAR1, OsYUC9 and OsYUC11 genes expressed highly in the endosperm cells (Figure 4a), suggesting these three genes might play important roles in controlling auxin biosynthesis in grains. However, only the expression of OsYUC11 was well correlated with the grain IAA content (Figure 4c). Based on these results, we hypothesize that OsYUC11 were mainly and especially responsible for the auxin biosynthesis in endosperm cells of rice and other auxin biosynthesis related genes may participate in separate signaling processes.

It is clear that the delay of caryopsis development and the lower starch accumulation and grain weight in $R G B 1$ knocking down lines are due to the lower auxin content in grains caused by the lower expression of OsYUC11 during grain filling stage. Furthermore, OsYUC11 also plays a positive regulatory role in the starch biosynthesis pathway by up-regulating the expression of several sucrose metabolism and starch biosynthesis related genes. Collectively, our study suggests that OsYUC11 is of crucial importance in regulating grain development and starch biosynthesis by controlling auxin content during grain filling stage. However, we still need direct evidence to verify that OsYUC11 is the key enzyme in controlling the level of IAA in rice grain. We tried to knock out OsYUC11 in WYJ8, but failed to obtain the regeneration plantlet from callus, likely because of disrupting the balance of auxin and cytokinin supplemented in medium. Next, we will modify the ratio of auxin and cytokinin in regeneration medium to create the null mutant of OsYUC11

In eukaryotes, transcription of genes is regulated by various transcription factors. Our results showed that OsYUC11 promoter may interact with several families of transcript factors, including MADS, MYB, CCAAT, etc. (Table S1), suggesting that the regulation of OsYUC11 expression is very complicated and involves signaling networks. However, if we considered the results of RNA-seq analysis that showed the differences of the expression of various transcription factors between $R G B 1 R i$ line and WYJ8 plant during grain filling stage, and the results of tissue-specific analysis of gene expression of these transcript factors, it is reasonable to assume that OsNF-YB1 might involve in the regulation of the expression of OsYUC11. Our present results provide biochemical evidence to support the conclusion that $O s N F-Y B 1$ was crucial importance in regulating OsYUC11 expression, and finally the auxin content in grains. OsNF-YB1 has been reported to activate the expression of sucrose transporters and waxy gene, and finally regulate the endosperms development. Knockout of OsNF-YB1 led to defective grains with chalky endosperms and significantly decreased grain weight (Bai et al. , 2016; Bello et al. , 2018). Our results provide a new insight that OsNF-YB1 regulates grain development not only through directly activating the sucrose metabolism and starch biosynthesis genes, but also through controlling the auxin accumulation.

In summary, according to our present results, a working model is proposed to illustrate the roles of RGB1 in regulating grain development and grain filling process. RGB1 may positively regulate $\backslash$ souts expression of transcription factor $O s N F-Y B 1$, which activates the $O s Y U C 11$ transcription by interacting with its promoter, and leads to an increase in auxin level. The increased auxin then stimulates the expression of sucrose metabolism and starch biosynthesis related genes in endosperm cells, as a consequence, the biosynthesis of starch and grain filling. However, the mechanism underlying how RGB1 regulates the expression of 
OsNF-YB1 remains to be further studied (Figure 6).

\section{Author contributions}

JSL and DPZ conceived the original idea and designed the overall project. DPZ and MYZ performed the experiments. JSL and DPZ interpreted all experimental results and collectively drafted the manuscript.

\section{Acknowledgements}

This work was founded by the Peacock Science and Technology Innovation Project of Shenzhen (KQJSCX20170328153821275), the National Science Foundation of China (31601230), the Key University Science Research Project of Jiangsu Province (17KJA210002), and Priority Academic Program Development of Jiangsu Higher Education Institutions. We thank Prof. Cunxu Wei and Dr. Juan Wang for the fruitful discussions and valuable suggestions on this study.

\section{Conflict of interest}

The authors declare no conflicts of interest.

\section{References}

Abu-Zaitoon, Y.M., Bennett, K., Normanly, J. and Nonhebel, H.M. (2012). A large increase in IAA during development of rice grains correlates with the expression of tryptophan aminotransferase OsTAR1 and a grain-specific YUCCA. Physiologia Plantarum, 146, 487-499. Ashikari, M., Wu, J., Yano, M., Sasaki, T. and Yoshimura, A. (1999). Rice gibberellin-insensitive dwarf mutant gene Dwarf 1 encodes the alpha-subunit of GTP-binding protein. Proceedings of the National Academy of Sciences of the United States of America, 96, 10284-10289. Bai, A., Lu, X., Li, D., Liu, J., Liu, C. (2016). NF-YB1-regulated expression of sucrose transporters in aleurone facilitates sugar loading to rice endosperm. Cell Research, 26, 384-388. Basunia, M. A. and Nonhebel, H. M. (2019). Hormonal regulation of cereal endosperm development with a focus on rice (Oryza sativa).Functional Plant Biology, 6, 493-506. Bello, B., Hou, Y., Zhao, J., Jiao, G., Wu, Y., Li, Z., Wang, Y., Tong, X., Wang, W., Yuan, W., Wei, X., Zhang, J. (2018). NF-YB1-YC12-bHLH144 complex directly activates Wx to regulate grain quality in rice (Oryza sativa L.). Plant Biotechnology Journal, 17, 1222-1235 Botella, J.R. (2012). Can heterotrimeric G proteins help to feed the world? Trends in Plant Science, 17, 563-68. Chen, C., Chen, H., Lin, Y., Shen, J., Shan, J., Qi, P., Shi, M., Zhu, M., Huang, X., Feng, Q., Han, B., Jiang, L., Gao., J. and Lin, H. (2014). A two-locus interaction causes interspecific hybrid weakness in rice. Nature Communications, 5, 3357. Figueiredo, D.D. and Köhler, C. (2018). Auxin: a molecular trigger of seed development. Genes and Development, 32, 479-490. Fujisawa, Y., Kato, T., Ohki, S., Ishikawa, A., Kitano, H., Sasaki, T., Asahi, T. and Iwasaki, Y. (1999). Suppression of the heterotrimeric G protein causes abnormal morphology, including dwarfism, in rice.Proceedings of the National Academy of Sciences of the United States of America, 96, 7575-7580. Ghorbani, J. M., Sorooshzadeh, A., Sanavy, S., Allahdadi, I. and Moradi, F. (2011). Effects of the exogenous application of auxin and cytokinin on carbohydrate accumulation in grains of rice under salt stress.Plant Growth Regulation, 65, 305-313. Hu, X., Qian, Q., Xu, T., Zhang, Y., Dong, G., Gao, T., Xie, Q, and Xue, Y. (2013). The U-box E3 ubiquitin ligase TUD1 functions with a heterotrimeric $\mathrm{G}$ alpha subunit to regulate Brassinosteroid-mediated growth in rice. PLoS Genetics, 9, e1003391. Huang, X., Qqian, Q., Liu, Z., Sun, H., He, S., Luo, D., Xia,G., Chu, C., Li, J. and $\mathrm{Fu}, \mathrm{X}$. (2009). Natural variation at the DEP1 locus enhances grain yield in rice. Nature Genetics, 41, 494-497. Jones, A. and Assmann, S. (2004). Plants: the latest model system for G-protein research. EMBO Reports, 5, 572-578. Keeling, P.L. and Myers, A.M. (2010). Biochemistry and genetics of starch synthesis. Annual Review of Food Science and Technology,1, 271-303. Li, N., Xu, R., Duan, P. and Li, Y. (2018). Control of grain size in rice. Plant Reproduction,https://doi.org/10.1007/s00497-018-0333-6 Li, Y., Fan, C., Xing, Y., Jiang, Y., Luo, L., Sun, L., Shao, D., Xu, C., Li, X., Xiao, J., He, Y. and Zhang, Q. (2011). Natural variation in GS5 plays an important role in regulating grain size and yield in rice. Nature Genetics, 43, 1266-69. Liang, J., Zhang, J. and Cao, X. (2001). Grain sink strength may be related to the poor grain filling of indica-japonica rice (Oryza sativa) hybrids. Physiologia Plantarum, 12, 470-477. Liu, L., Tong, H., Xiao, Y., Che, R., Xu, F., Hu, B., Liang, C., Chu, J., Li, J. and Chu, C. (2015). Activation of 
Big Grain1 significantly improves grain size by regulating auxin transport in rice.Proceedings of the National Academy of Sciences of the United States of America, 112, 11102-11107. Liu, Q., Han, R., Wu, K., Zhang, J., Ye, Y., Wang, S., Chen, J., Pan, Y., Li, Q., Xu, X., Zhou, J., Tao, D., Wu, Y. and Fu, X. (2018), G protein betagamma subunits determine grain size through interaction with MADS-domain transcription factors in rice. Nature Communications,9, 852. Lü, B., Guo, Z. and Liang, J. (2008). Effects of the activities of key enzymes involved in starch biosynthesis on the fine structure of amylopectin in developing rice (Oryza sativa L.) endosperms.Science in China. Series C, 51, 863-871. Mao, H., Sun, S., Yao, J., Wang, C., Yu, S., Xu, C., $\mathrm{Li}$, X. and Zhang, Q. (2010). Linking differential domain functions of the GS3 protein to natural variation of grain size in rice. Proceedings of the National Academy of Sciences of the United States of America, 107, 19579-19584. McAdam, E. L, Meitzel, T., Quittenden, L.J., Davidson, S.E., Dalmais, M., Bendahmane, A.I., Thompson, R., Smith, J.J., Nichols, D.S., Urquhart, S., Gélinas-Marion, A., Aubert, G. and Ross, J.J. (2017). Evidence that auxin is required for normal seed size and starch synthesis in pea. New Phytologists, 216,193204. Miao, J., Yang, Z., Zhang, D., Wang, Y., Xu, M., Zhou, L., Wang, J., Wu, S., Yao, Y., Du, X., Gu, F., Gong, Z., Gu, M., Liang, G. and Zhou, Y. (2018). Mutation of RGG2, which encodes a type B heterotrimeric $\mathrm{G}$ protein gamma subunit, increases grain size and yield production in rice. Plant Biotechnology Journal, 17, 650-664. Mortazavi, A., Williams, B. A., McCue, K., Schaeffer, L. and Wold, B. (2008). Mapping and quantifying mammalian transcriptomes by RNA-Seq. Nature Methods, 5, 621-28. Ohdan, T., Francisco, Jr. P., Sawada, T., Hirose, T., Terao, T., Satoh, H. and Nakamura,Y. (2005). Expression profiling of genes involved in starch synthesis in sink and source organs of rice. Journal of Experimental Botany, 56, 3229-3244. Oki, K., Fujisawa, Y., Kato, H. and Iwasaki, Y. (2005). Study of the constitutively active form of the alpha subunit of rice heterotrimeric G proteins. Plant Cell Physiology, 46, 381-386. Sun, S., Wang, L., Mao, H., Shao, L., Li, X., Xiao, J., Ouyang, Y. and Zhang, Q. (2018). A G protein pathway determines grain size in rice.Nature Communications, 9, 851. Tamaki, H., Reguera, M., Abdel-Tawab, Y.M., Takebayashi, Y., Kasahara, H. and Blumwald, E. (2015). Targeting hormone-related pathways to improve grain yield in rice: a chemical approach. PLoS One, 10, e0131213.doi:10.1371/journal.pone.0131213 Tang, T., Xie, H., Wang, Y., Lü, B. and Liang, J. (2009). The effect of sucrose and abscisic acid interaction on sucrose synthase and its relationship to grain filling of rice (Oryza sativa L.).Journal of Experimental Botany, 60, 2641-2652. Tetlow, I., Morell, M. and Emes, M. (2004). Recent developments in understanding the regulation of starch metabolism in higher plants.Journal of Experimental Botany, 55, 2131-2145. Trapnell, C., Roberts, A., Goff, L., Pertea, G., Kim, D., Kelley, D.R., Pimentel, H., Salzberg, S.L., Rinn, J.L. and Pachter, L. (2012). Differential gene and transcript expression analysis of RNA-Seq experiments with TopHat and Cufflinks. Nature Protocol, 7, 562-578. Trusov, Y., Chakravorty, D. and Botella, J.R. (2012). Diversity of heterotrimeric G-protein gamma subunits in plant. BMC Research Notes, 5: 608. Urano, D. and Jones, A.M. (2014). Heterotrimeric G proteincoupled signaling in plants. Annual Review of Plant Biology, 65, 365-384. Urano, D., Chen, J.G., Botella, J.R. and Jones, A.M. (2013). Heterotrimeric G protein signalling in the plant kingdom. Open Biology, 3, 120186. Utsunomiya, Y., Samejima, C., Takayanagi, Y., Izawa, Y., Yoshida, T., Sawada, Y., Fujisawa, Y., Kato, H. and Iwasaki, Y. (2011). Suppression of the rice heterotrimeric G protein beta-subunit gene, RGB1, causes dwarfism and browning of internodes and lamina joint regions.Plant Journal, 67, 907-916. Wang, Z., Chen, C., Xu, Y., Jiang, R., Han, Y., Xu, Z. and Chong, K. (2004). A practical vector for efficient knockdown of gene expression in rice (Oryza sativa L.). Plant Molecular Biology Reporter,22, 409-417. Yang, J. and Zhang, J. (2010). Grain-filling problem in 'super' rice.Journal of Experimental Botany, 61, 1-5. Yang, J., Zhang, J., Wang, Z., Liu, K. and Wang, P. (2006). Post-anthesis development of inferior and superior spikelets in rice in relation to abscisic acid and ethylene. Journal of Experimental Botany, 57, 149-160. Zeeman, S.C., Kossmann, J. and Smith, A.M. (2010) Starch: its metabolism, evolution, and biotechnological modification in plants. Annual Review of Plant Biology, 61, 209-234. Zhang T, Xu P, Wang W, Wang S, Caruana JC, Yang H-Q and Lian H (2018). Arabidopsis G-protein subunit AGB1 interacts with BES1 to regulate brassinosteroid signaling and cell elongation. Frontiers in Plant Science, 8, 2225. Zhang, C., Bai, M. and Chong, K. (2014). Brassinosteroid-mediated regulation of agronomic traits in rice. Plant Cell Reproduction,33, 683-696. Zhang, D., Xu, W., Chen, J. and Liang, J. (2019). Rice heterotrimeric G protein $\gamma$ subunit, qPE9-1, regulates development of endosperm cells and starch accumulation of grains. Rice, 12:91 https://doi.org/10.1186/s12284-019-0344-4. Zhao, Y. (2008). The role of local biosynthesis of auxin and 
cytokinin in plant development. Current Opinion on Plant Biology, 11, 16-22. Zhao, Z., Zhang, Y., Liu, X., Zhang, X., Liu, S., Yu, X., Ren, Y., Zheng, X., Zhou, K., Jiang, L., Guo, X., Gai, Y.,Wu,C., Zhai, H., Wang, H. and Wan, J. (2013). A role for a dioxygenase in auxin metabolism and reproductive development in rice. Developmenttal Cell, 27, 113-122. Zhou, Y., Zhu, J., Li, Z., Yi, C., Liu, J., Zhang, H., Tang, S., Gu, M. and Liang, G. (2009). Deletion in a quantitative trait geneqPE9-1 associated with panicle erectness improves plant architecture during rice domestication. Genetics, 183, 315-324. Zhu, T., Budworth, P., Chen, W., Provart, N., Chang, H., Guimil, S., Su, W., Estes, B., Zou, G. and Wang, X. (2003). Transcriptional control of nutrient partitioning during rice grain filling. Plant Biotechnology Jornal, 1, 59-70 Zuo, J. and Li, J. (2014) Molecular genetic dissection of quantitative trait loci regulating rice grain size. Annual Review of Genetics,48, 99-118.

Figure legends

Figure 1. Comparison of grain size and dry weight accumulation between wildtype (WYJ8) plant and RGB1 RNA-interfered transgenic lines.

(a) Immunoblot analysis of the transgenic plants. Total proteins are extracted from the panicles, and $10 \mu \mathrm{g}$ protein was used for western blotting. (b) Grain morphology (size and shape). Scale bar: $15 \mathrm{~mm}$. (c-f) Comparison of grain size and weight between wild type (WYJ8) and RGB1 RNA-interfered transgenic lines:(c) Grain length; (d) Grain width; (e) Grain thickness; (f) 1000-grain weight. (g) The grain starch content. (h) The changes of caryopsis morphology at different days after flowering. (i) Changes of grain weight during grain-filling. Data are shown as means \pm SDs $(n=15)$.

Figure 2. Expressional profiles of sucrose metabolism and starch synthesis related genes during grain filling stage of $W Y J 8$ plant and RGB1Ri lines

(a) Heat maps of differentially expressed genes (DGEs) involved in the starch biosynthesis. The maps were plotted using the value of RPKM for each gene in the different samples: blue indicates low values, red indicates high values. The indicated scale is the $\log 2$ value of the normalized level of gene expression. (b) qRT-PCR analysis showing the changes of genes encoding sucrose metabolism and starch biosynthesis related enzymes during grain filling (from 5 to $25 \mathrm{DAF}$ ).(c) Western blotting assay showing the changes of the levels of several enzymes/proteins related to starch biosynthesis at the early stage of grain filling ( 5 and 10 DAF).

Figure 3. Expressional profiles of auxin biosynthesis related genes and changes of IAA in grains of $W Y J 8$ plant and RGB1Ri line and effects of application of NAA on starch biosynthesis

(a) Heat maps of differentially expressed genes (DGEs) involved in auxin biosynthesis. The maps were plotted using the value of RPKM for each gene in the different samples: blue indicates low values, red indicates high values. The indicated scale is the log2 value of the normalized level of gene expression. (b) Changes of IAA level in rice endosperm cells of different genotypes during grain filling stage. (c) Effects of exogenous auxin (NAA) application on starch accumulation of grains of different genotypes. (d)Effects of exogenous auxin (NAA) application on grain weight of different genotypes. (e) Effects of exogenous auxin (NAA) application on the expression of genes related to sucrose metabolism and starch biosynthesis at 10 DAF. Data are shown as means \pm SDs $(n=5)$.

Figure 4. Tissue-specific expressions of $O s Y U C 11$ and its relation to auxin content of grains

(a) Expression analysis of $O s Y U C 11$ in public microarray data. The maps were plotted using the value of normalized signal intensity for each gene in the different samples: blue indicates low values, red indicates high values. The indicated scale is the $\log 2$ value of the normalized level of gene expression. (b) qRT-PCR analysis showing the expression of $O s Y U C 11$ in the grains of WYJ8 and $R G B 1 R i$ lines during filling stage.

(c)The relationship between the expressional levels of $O s Y U C 11$ and IAA content in grains.

Figure 5. The transcript factor OsNF-YB1 directly interacts with promoter of $O s Y U C 11$

(a) Venn diagram showing number of genes included in co-expressed with OsYUC11 and putative transcript factors which bind to the promoter of OsYUC11. Venn diagram has been generated using Venn 
(http://bioinfogp.cnb.csic.es/tools/venny/index.html).(b) Heat map of the expression of candidate transcript factors in WYJ8 and RGB1Ri line during filling stage. (c)Expression of OsNF-YB1 in WYJ8 and $R G B 1 R i$ lines.(d) Correlation between the expression of $O s Y U C 11$ and $O s N F-Y B 1$. (e) EMSA assay showing OsNF-YB1 binding to the OsYUC11 promoter in vitro .

\section{Figure 6. A working model on RGB1 regulation on grain filling}

\section{Supporting Information}

Table S1 The quantitative trait loci (QTLs)/genes to control grain size and grain filling.

Table S2 Predicted transcript factors binding to the promoter of OsYUC11.

Figure S1 Transcriptome comparison between developing grains of the wild type ( WYJ8) and RGB1 RNAinterfered transgenic line (RGB1Ri-5 ) using RNA-SEQ. (a) Numbers of genes up- and down-regulated in the comparisons between WYJ8 andRGB1Ri-5 at 5 and 10 day after flowering (DAF). (b) The top 5 enriched KEGG pathways in grains.

Figure S2 Heat maps of differentially expressed genes (DGEs) involved in auxin transport (a), auxin biosynthesis(b) and auxin signaling (c). The maps were plotted using the value of RPKM for each gene in the different samples: blue indicates low values, red indicates high values. The indicated scale is the $\log 2$ value of the normalized level of gene expression.

Figure S3 Expressions of OsTAR1 and OsYUC9 in WYJ8 and RGB1Ri lines during filling stage (a), and its relation to auxin content of grains (b).
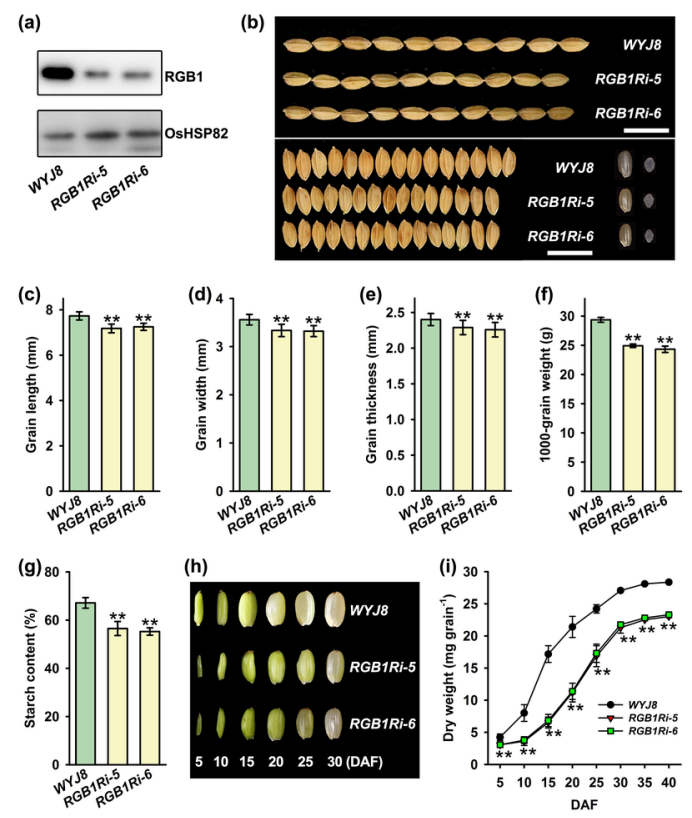

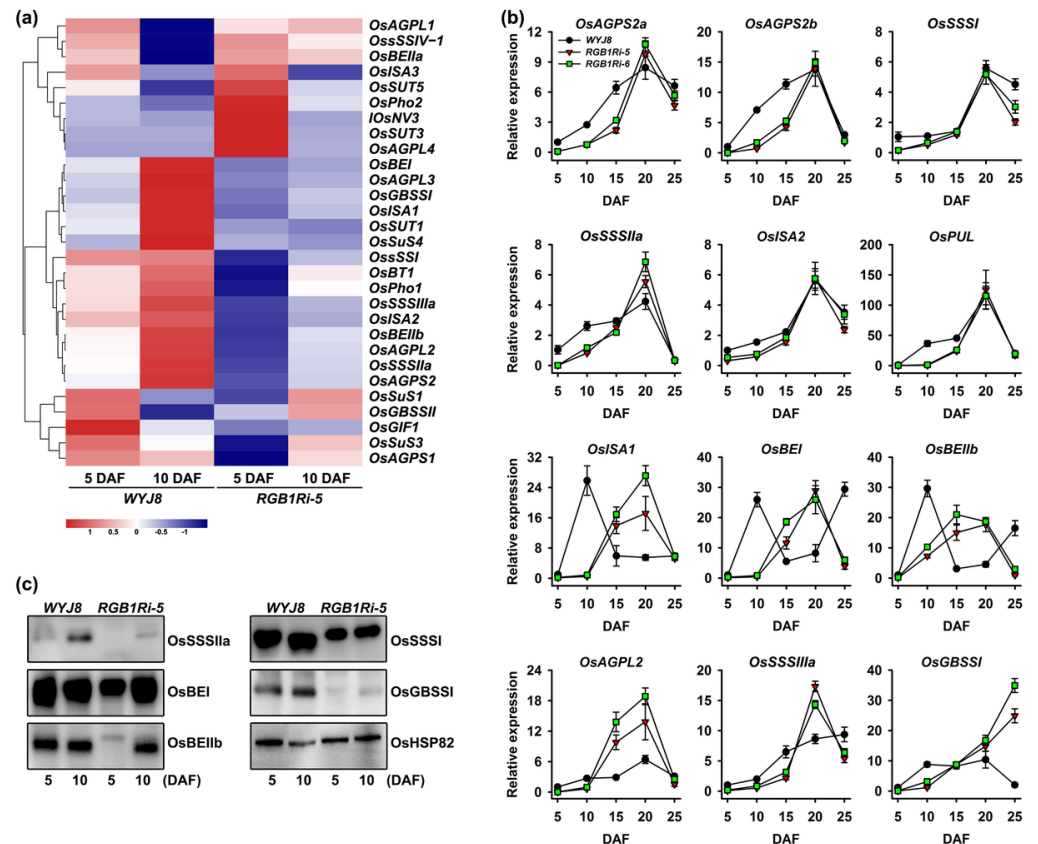

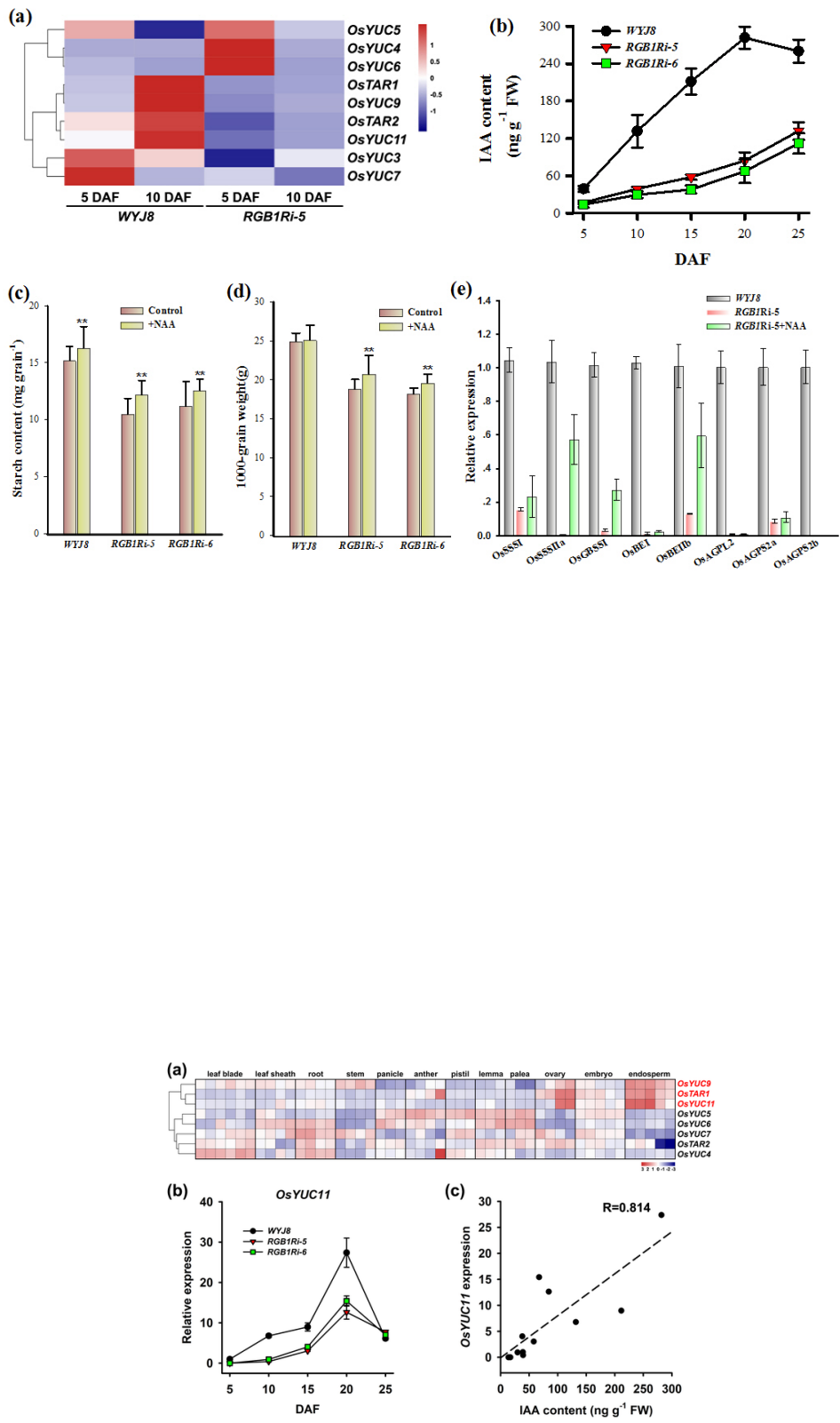
(a)
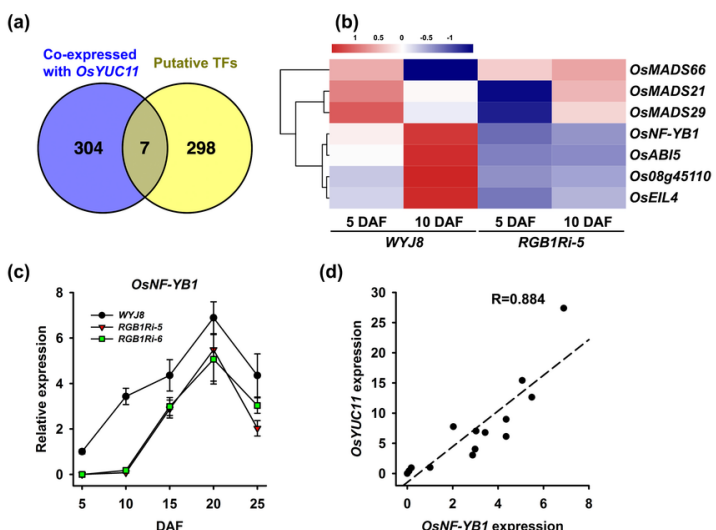

(d)

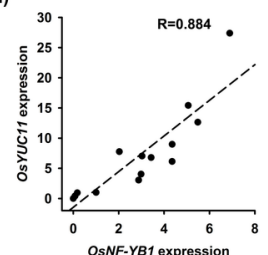

(e)

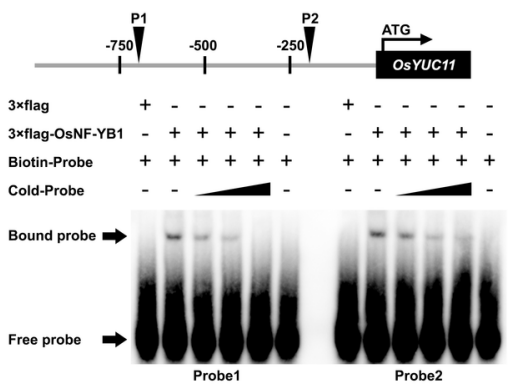




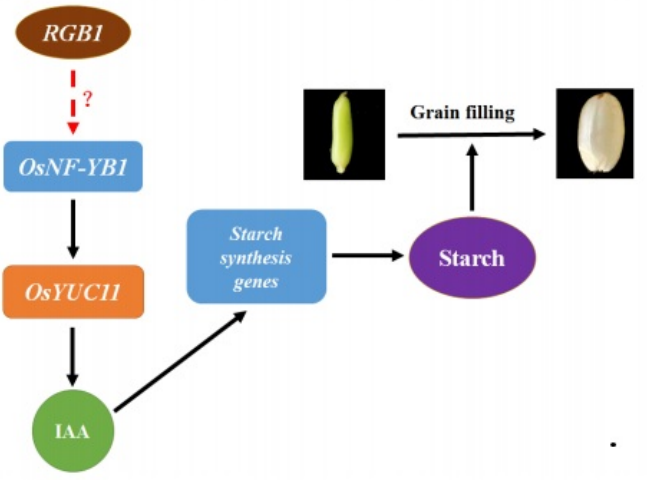

